\title{
Surface treatment on cobalt and titanium alloys using picosecond laser pulses in burst mode
}

\author{
Daniel Metzner $^{1}$ (D) $\cdot$ Peter Lickschat $^{1} \cdot$ Steffen Weißmantel ${ }^{1}$
}

Received: 21 August 2020 / Accepted: 28 November 2020 / Published online: 30 December 2020

(c) The Author(s) 2020

\begin{abstract}
The authors report on the results of surface treatment experiments using a solid-state amplified laser source emitting laser pulses with a pulse duration of $10 \mathrm{ps}$. The laser source allows the generation of pulse trains (bursts) with an intra-burst pulse repetition rate of $80 \mathrm{MHz}$ (pulse-to-pulse time interval about $12.5 \mathrm{~ns}$ ) with up to eight pulses per burst. In this study a wavelength of $1064 \mathrm{~nm}$ was used to investigate both ablation of material and laser-induced surface modifications occuring in metallic implant alloys CoCrMo (cobalt-chromium-molybdenum) and TiAlV (titanium-aluminum-vanadium) in dependence of the number of pulses and fluences per pulse in the burst. By using the burst mode, a smoothing effect occurs in a certain parameter range, resulting in very low surface roughness of the generated microstructures. It is demonstrated that at fluences per pulse which are smaller than the material-specific ablation threshold, a self-organized pore formation takes place if a defined number of pulses per burst is used. Thus, the advantage of the MHz burst mode in terms of a possible surface modification is established.
\end{abstract}

Keywords Ultrashort laser pulses $\cdot$ High-frequency $\cdot$ Burst mode $\cdot$ Surface modification $\cdot$ Pore formation

\section{Introduction}

The structuring and texturing of material surfaces using ultrashort pulsed laser radiation is a technology allowing the production of nano- to micrometer-sized structures by ablation with a considerable control of different surface properties. These structures can be used to control several functionalities, including optical properties, hydrophobicity $[1,2]$, oleophobia $[3,4]$ and even the growth of biological material on the surface [5, 6]. Each specific surface functionality requires its own modification of the material surface and yielding appropriate physical and chemical properties of the surface material after the irradiation. Currently available laser beam sources with pulse durations in the femto- and picosecond regime provide sufficient intensity for ablating, shaping or modifying a wide range of different materials, including stainless steel and biological tissue.

By using ultrashort pulsed laser radiation at fluences above the material-specific ablation threshold, material is ablated, resulting in a characteristic surface structure. These laser-induced periodic surface structures (LIPSS) [7, 8] form regular patterns and mainly occur with ultrashort laser pulses and fluences near the ablation threshold $[9,10]$. These structures can change the wettability characteristics of the material surface, as well as optical and tribological properties. Heat accumulation, caused by very high pulse repetition frequencies, has been avoided as far as possible during the generation of LIPPS $[11,12]$.

In this paper the authors present a study on metallic implant material that focuses on the influence of pulse trains (bursts) with an intra-burst pulse repetition frequency of $80 \mathrm{MHz}$ on heat accumulation induced surface modification. Furthermore, the aim of the study was to investigate the possibility of adjusting the surface morphology by a systematic variation of the number of pulses and fluence per pulse in the burst. The results of this study are supposed to evaluate possible fields of applications, which are based on the surface modification using ultrashort pulses in the burst mode.

Daniel Metzner

metzner@hs-mittweida.de

1 University of Applied Sciences, Technikumplatz 17,

09648 Mittweida, Germany 


\section{Materials and methods}

A solid state laser (Fuego, Time Bandwidth Products) with a pulse duration of $10 \mathrm{ps}$ and an emitting wavelength of $1064 \mathrm{~nm}$ was used for the experiments. The laser can generate pulse trains (bursts) with a certain number of pulses $N$ ranging from one (single pulse mode) up to eight in the $\mathrm{MHz}$ burst mode (Fig. 1). The intra-burst pulse repetition frequency was $80 \mathrm{MHz}$, which corresponds to the time delay $t_{\mathrm{p}-\mathrm{p}}$ between subsequent pulses of about $12.5 \mathrm{~ns}$.

The spatial intensity distributions nearly equals an ideal spatial gaussian profile (diffraction coefficient $M^{2}<$ 1.1). A spherical lens with a focal length of $100 \mathrm{~mm}$ (Sill Optics) was used for focusing the laser radiation (wavelength $1064 \mathrm{~nm}$ ) to a beam waist radius of $14.1 \mu \mathrm{m}$ on the material surface. With ten unidirectional scan levels (Fig. 2) and a tracked focus position, square structures with an edge length $l$ of $500 \mu \mathrm{m}$ and a geometric burst

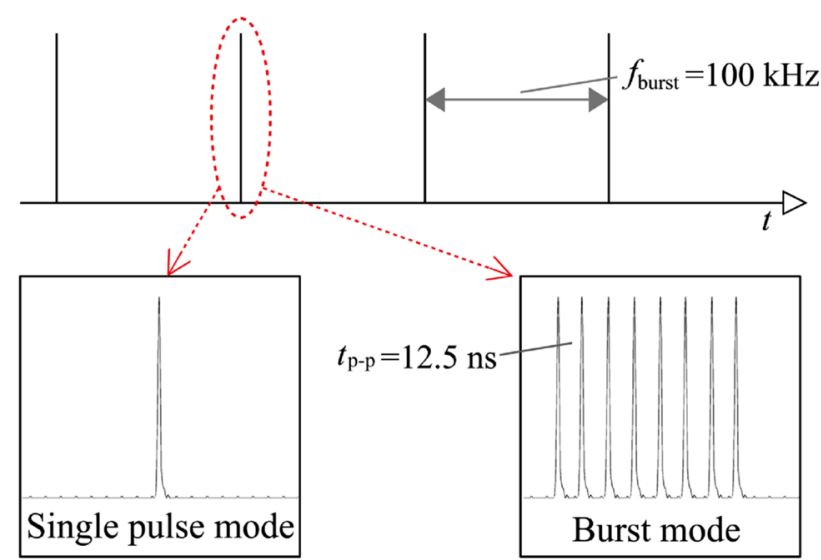

Fig. 1 Schematic representation of the burst mode in comparison to the single pulse mode [13]

$d x$ and line hatch $d y$ of $6 \mu \mathrm{m}$, were ablated in CoCrMo

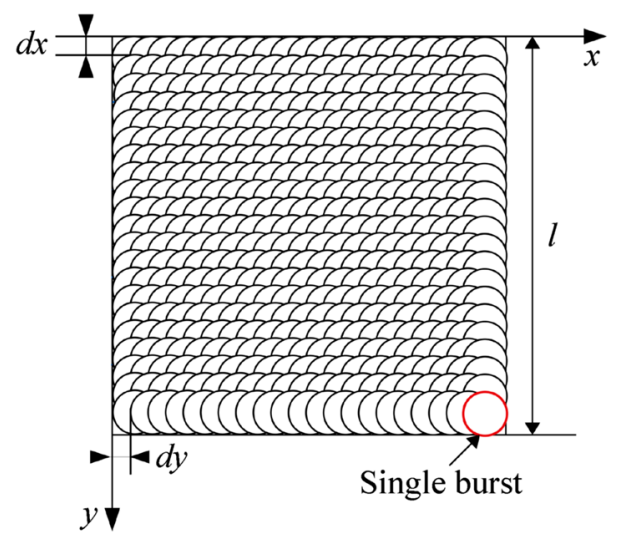

Fig. 2 Schematic representation of the generated structures [13] and TiAlV. The experiments were conducted in air at a constant ambient pressure. Using a burst repetition rate of $100 \mathrm{kHz}$ and a burst hatch of $6 \mu \mathrm{m}$ resulted in a scan speed of $0.6 \mathrm{~m} / \mathrm{s}$.

The applied fluences in the range between 0.2 and 0.75 $\mathrm{J} / \mathrm{cm}^{2}$ per pulse in a burst were chosen to ensure that the absorbed laser radiation is smaller but also larger than the ablation threshold of the investigated materials [13, 14]. Experiments at fluences per pulse above the ablation threshold are designated in this paper as "ablation regime" and the fluences below the ablation threshold as "non-ablation regime". In this study only the heat accumulation due to the high intra-burst pulse repetition frequency will be considered and discussed. To avoid heat accumulation due to multiburst laser ablation [15], a relatively low burst repetition frequency $f_{\text {burst }}$ of $100 \mathrm{kHz}$ was preferred for the experiments performed in this study. The dimensions of the produced structures as well as the structure depth $z$ and the surface roughness $S_{\mathrm{a}}$ have been determined by confocal laser scanning microscopy (Olympus model Lext 3D OLS4100). The energy dispersive X-ray analysis (EDX) and further image recordings were performed by scanning electron microscopy (SEM) (Jeol model JSM-6512V). For a better visualization of the results, the data points in the presented figures in this work are interpolated with a cubic spline function and plotted as dashed lines.

Two materials are investigated, CoCrMo (cobald chromium molybdenum) and TiAlV (titanium aluminum vanadium) alloy that meets the chemical and mechanical requirements of ISO 5832 and ASTM F1537 for CoCrMo and ASTM F136 for TiAlV. To ensure a homogeneous diffusion of the laser-induced heat, all investigated material samples have a thickness of $5 \mathrm{~mm}$. Furthermore, for an accurate measurement of the resulting surface roughness $S_{\mathrm{a}}$, the material surface is polished to $S_{\mathrm{a}}=10 \mathrm{~nm}$ prior to the structuring.

\section{Burst mode induced heat accumulation}

Due to the short intra-burst pulse repetition time of $12.5 \mathrm{~ns}$, the laser-induced heat induced by each laser pulse in the interaction zone cannot completely dissipate within the material before the subsequent pulse interacts with the material surface $[13,16]$. As a result, heat is accumulated and the temperature in the interaction zone increases with an increase in the number of pulses in the burst. Above a certain number of pulses the melting temperature can be exceeded by heat accumulation and a melt film is formed. This effect can occur in the ablation and in the non-ablation regime. By using an eight-pulse burst with an intra-burst pulse repetition time of $12.5 \mathrm{~ns}$, the burst has a time width of $87.5 \mathrm{~ns}$. In conclusion, melting films can be formed which, depending on the number of pulses and fluence per pulse in the burst, 
remain for a period of a few picoseconds to over $100 \mathrm{~ns}$ until they solidify $[13,16]$. The movement within the melt film is described in terms of melt convection [17, 18]. Free convection describes, thereby, the movement within the melt film without external impact, which is influenced by the surface tension induced by the temperature gradient, also called the Marangoni effect, and the depth of the melt film [19, 20]. Due to the Marangoni effect, bubbles can be formed within the melt film which can be stabilized to a foam-like structure $[21,22]$. In the case of a forced convection, an external force (e.g. the ablation pressure of the pulse or the boiling vapour pressure in the case of evaporation) influences the motion of the melt film [23]. In conclusion, different surface structures caused by the dynamics of the melt film can be produced. These melting dynamics can induce a smoothing effect to obtain structured surfaces with a very low roughness [13, $24,25]$. In the case of a further increase of the surface temperature by accumulated heat, the boiling temperature can be exceeded even in the non-ablation regime and material can be removed by evaporation [16].

\section{Results and discussion}

\subsection{Ablation depth}

With the determined ablation depths on CoCrMo, presented in Fig. 3, it is apparent that at a fluence of $0.25 \mathrm{~J} / \mathrm{cm}^{2}$ per pulse, a structure depth is first measurable starting from the sixth pulse and at $0.5 \mathrm{~J} / \mathrm{cm}^{2}$ from the fifth pulse in the burst.

Both fluences are smaller than the ablation threshold of CoCrMo [13] and thus in the non-ablation regime. Due to the high intra-burst pulse repetition rate of $80 \mathrm{MHz}$, residual heat is accumulated, which exceeds the boiling temperature in this regime after a certain number of pulses and material is removed by evaporation. At a fluence of $0.75 \mathrm{~J} / \mathrm{cm}^{2}$, the

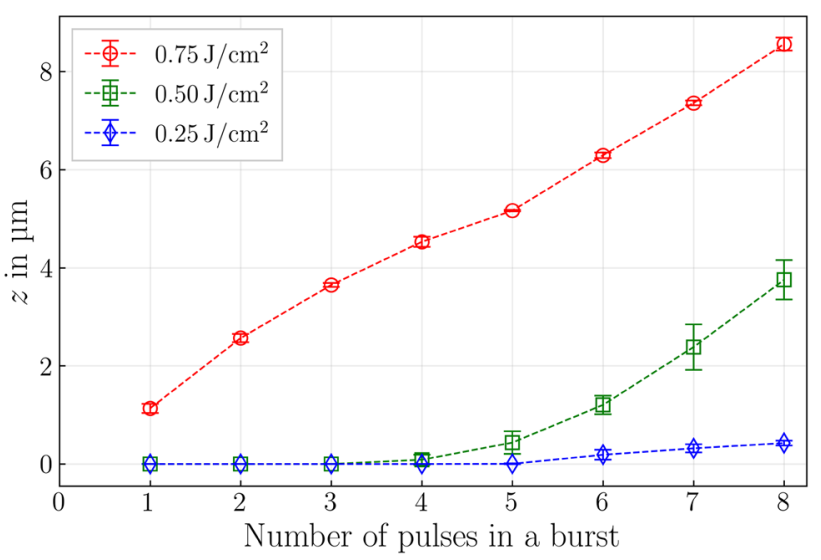

Fig. 3 Structure depth of the machined CoCrMo as a function of the fluence and the number of pulses in the burst

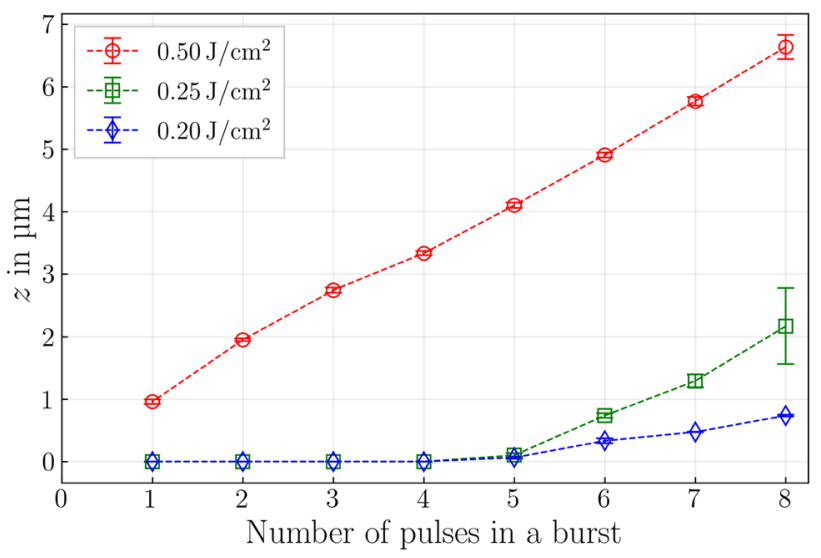

Fig. 4 Structure depth of the machined TiAlV as a function of the fluence and the number of pulses in the burst

ablation threshold is exceeded with the first pulse (ablation regime) in the burst which explains the much deeper structures compared to $0.25 \mathrm{~J} / \mathrm{cm}^{2}$ and $0.5 \mathrm{~J} / \mathrm{cm}^{2}$.

For the determined structure depths on TiAlV,

illustrated in Fig. 4, a similar ablation behaviour can be observed compared to those on CoCrMo. The threshold fluence of TiAlV is slightly lower compared to CoCrMo [14], resulting in an ablation of material at a fluence of $0.5 \mathrm{~J} / \mathrm{cm}^{2}$ starting with the first pulse in the burst (ablation regime). At fluences in the non-ablation regime, such as $0.25 \mathrm{~J} / \mathrm{cm}^{2}$ and $0.2 \mathrm{~J} / \mathrm{cm}^{2}$, material is removed by accumulated residual heat induced evaporation each starting with the sixth pulse.

\subsection{Surface roughness and pore formation}

The surface roughness $S_{\mathrm{a}}$ of the structure bottoms as a function of the number of pulses and of the fluence per pulse in the burst have been measured with confocal laser scanning microscopy and are visualized in this section. Furthermore, for a qualitative estimation of the obtained surface structures, SEM images of the treated structure bottoms are presented.

Based on the determined surface roughness on $\mathrm{CoCrMo}$, shown in Fig. 5, it is evident that different values of surface roughness

can be obtained by using the burst mode depending on the number of pulses in the burst and on the fluence per pulse. Considering the surface roughness of the untreated bulk material of $10 \mathrm{~nm}$, an increase of the surface roughness to approx. $150 \mathrm{~nm}$ is observed for all investigated fluences in the single pulse mode (one pulse per burst). The SEM images in Fig. 6 for $0.25 \mathrm{~J} / \mathrm{cm}^{2}$, Fig. 7 for $0.5 \mathrm{~J} / \mathrm{cm}^{2}$, and Fig. 8 for $0.75 \mathrm{~J} / \mathrm{cm}^{2}$ illustrate that the increase in the surface roughness in the single pulse mode is caused by generated LIPPS. In the ablation regime $\left(0.75 \mathrm{~J} / \mathrm{cm}^{2}\right)$, an increase in the number of pulses in the burst generates a melt film in 


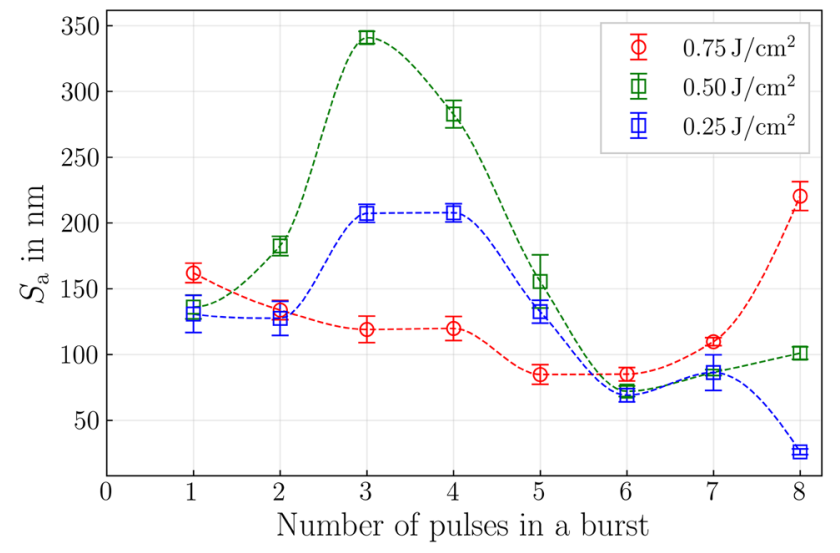

Fig. 5 Surface roughness of the irradiated areas on CoCrMo as a function of the fluence and of the number of pulses in the burst the interaction zone as a result of the accumulated heat. The Marangoni effect and the convection forced by the ablation pressure leads to a smoothing effect, which reduces the surface roughness to $90 \mathrm{~nm}$ up to the sixth pulse in the burst. The corresponding SEM images in Fig. 8 illustrate that the LIPSS are flattened.

With a further increase in the number of pulses, the melt film gets deeper and as a result of the melt dynamics, melt is pressed out of the interaction zone and solidifies as bulge volume $[13,26]$. This can be seen in Fig. 8 with the eighth pulse in the burst. In consequence, the surface roughness increases with the seventh and eighth pulse in the burst.

In the non-ablation regime, the surface roughness, shows in Fig. 5, indicates a considerably different dependence on the number of pulses in the burst compared to the ablation regime. When using a higher number of pulses in the burst,

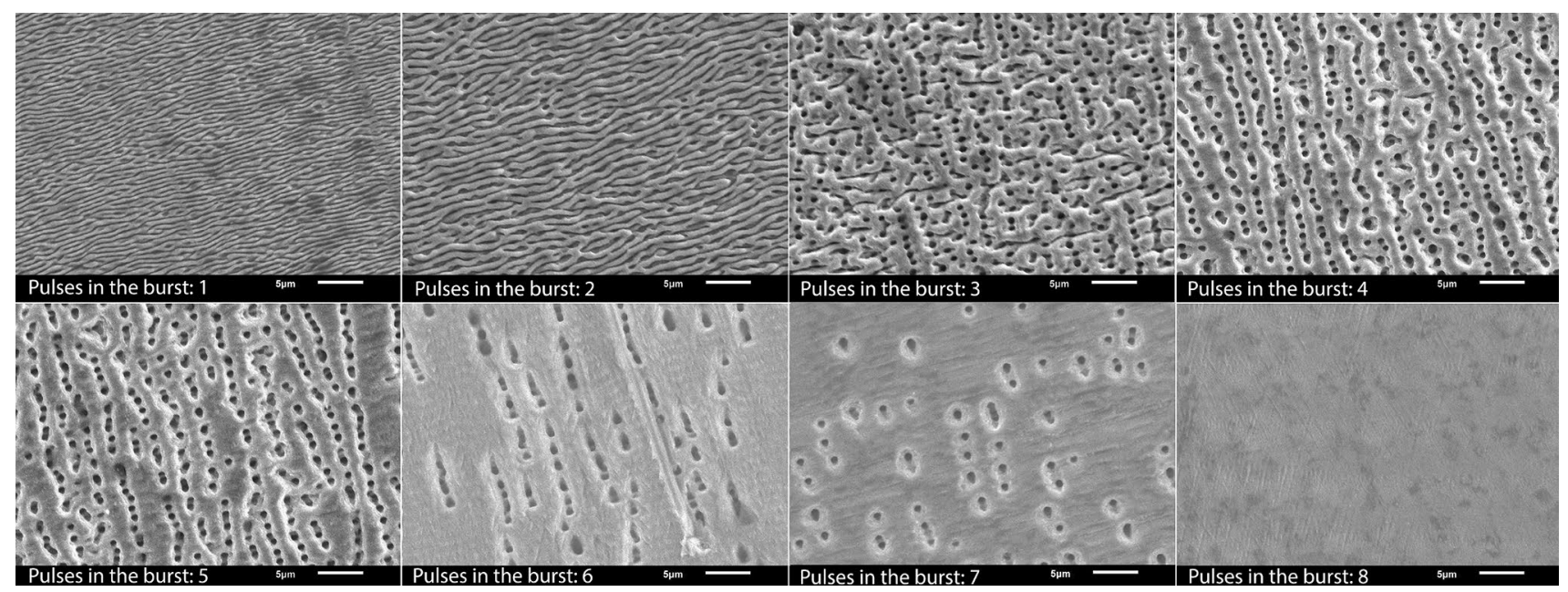

Fig. 6 SEM images of the irradiated CoCrMo surface as a function of the number of pulses in the burst at a fluence of $0.25 \mathrm{~J} / \mathrm{cm}^{2}$ per pulse

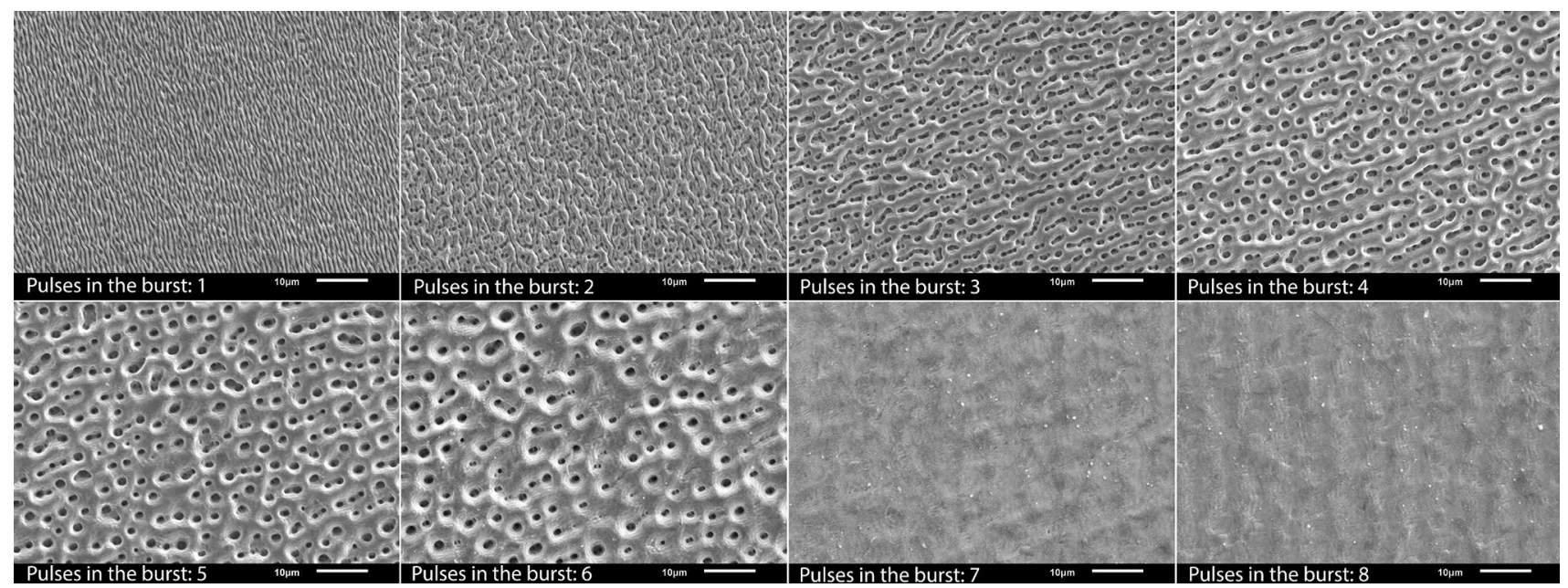

Fig. 7 SEM images of the irradiated CoCrMo surface as a function of the number of pulses in the burst at a fluence of $0.5 \mathrm{~J} / \mathrm{cm}^{2}$ per pulse 


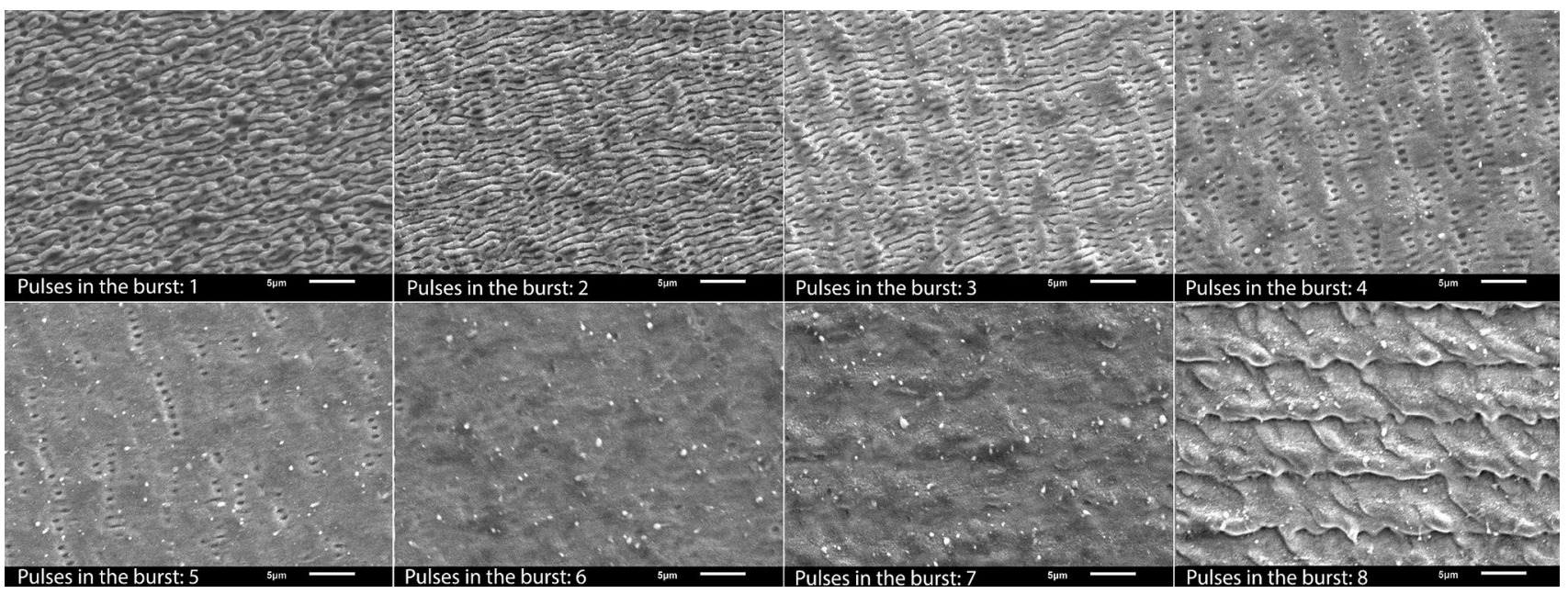

Fig. 8 SEM images of the irradiated CoCrMo surface as a function of the number of pulses in the burst at a fluence of $0.75 \mathrm{~J} / \mathrm{cm}^{2}$ per pulse

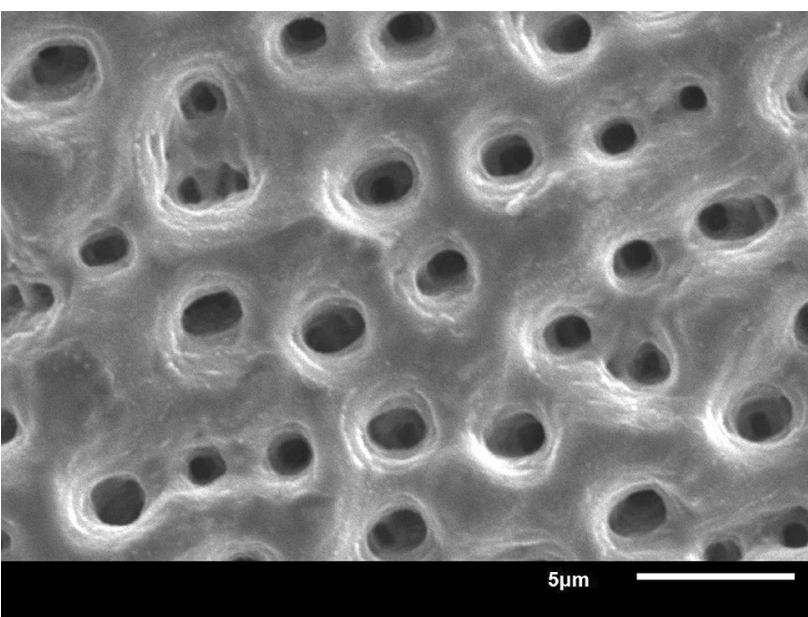

Fig. 9 Pore formation on CoCrMo alloy with four pulses in burst and a fluence of $0.5 \mathrm{~J} / \mathrm{cm}^{2}$ per pulse

the surface roughness increases up to the fifth pulse in the burst compared to the single pulse mode. As can be seen in Fig. 6 for $0.25 \mathrm{~J} / \mathrm{cm}^{2} \mathrm{~J} / \mathrm{cm}^{2}$ and in Fig. 7 for $0.5 \mathrm{~J} / \mathrm{cm}^{2}$, the LIPPS generated in the single pulse mode is modified by the melt convection to a self-organizing pore structure. The pore structure which is illustrated in Fig. 9 demonstrates that the pores are not only a formation of pits on the surface of the material, but rather a foam structure which extends into the depth.

The surface roughness presented in Fig. 5 directly compared with the corresponding structure depths from Fig. 3 proves that the pore formation is most pronounced if no material is removed by evaporation in the non-ablation regime. The pores disappear starting from the sixth pulse in the burst, induced by the boiling vapour pressure (Figs. 6,7).

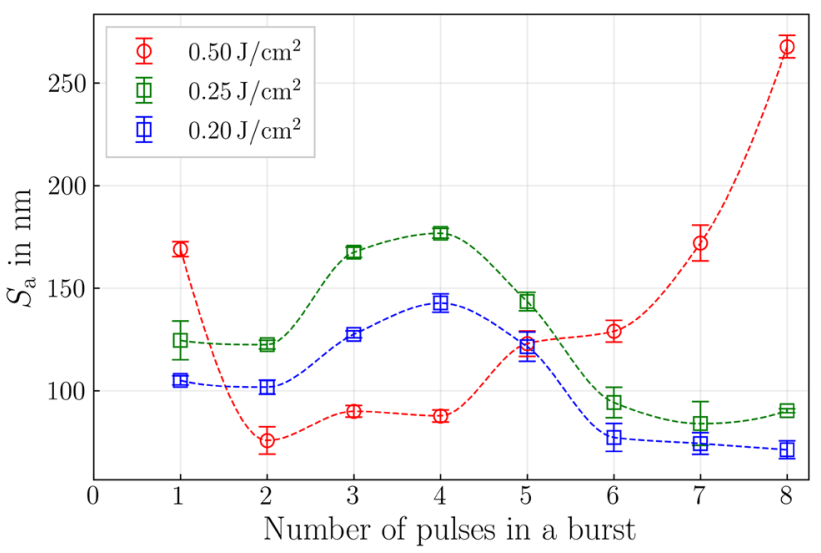

Fig. 10 Surface roughness of the irradiated areas on TiAlV as a function of the fluence and of the number of pulses in the burst

In the case of TiAlV, the measured surface roughness, shown in Fig. 10, indicates a similar trend as the resulting roughness of $\mathrm{CoCrMo}$. The surface roughness of the untreated material of $10 \mathrm{~nm}$ increases up to $160 \mathrm{~nm}$ due to the generation of LIPPS in the single pulse mode. The corresponding SEM images are illustrated in Fig. 11 for $0.2 \mathrm{~J} /$ $\mathrm{cm}^{2}$, Fig. 12 for $0.25 \mathrm{~J} / \mathrm{cm}^{2}$, and Fig. 13 for $0.5 \mathrm{~J} / \mathrm{cm}^{2}$. In the ablation regime $\left(0.5 \mathrm{~J} / \mathrm{cm}^{2}\right)$ the LIPPS are smoothed by the melt dynamics, resulting in a reduction of surface roughness up to the sixth pulse (compared to the single pulse mode). By using a higher number of pulses in the burst, the surface roughness increases due to the bulge volume generated with the seventh and eighth pulse.

In the non-ablation mode, the surface roughness increases with a higher number of pulses and reaches a maximum with the fourth pulse as shown in Fig. 10. This maximum can be explained on the basis of Figs. 11, 12 to the effect that the generated pores reach their maximum dimension 


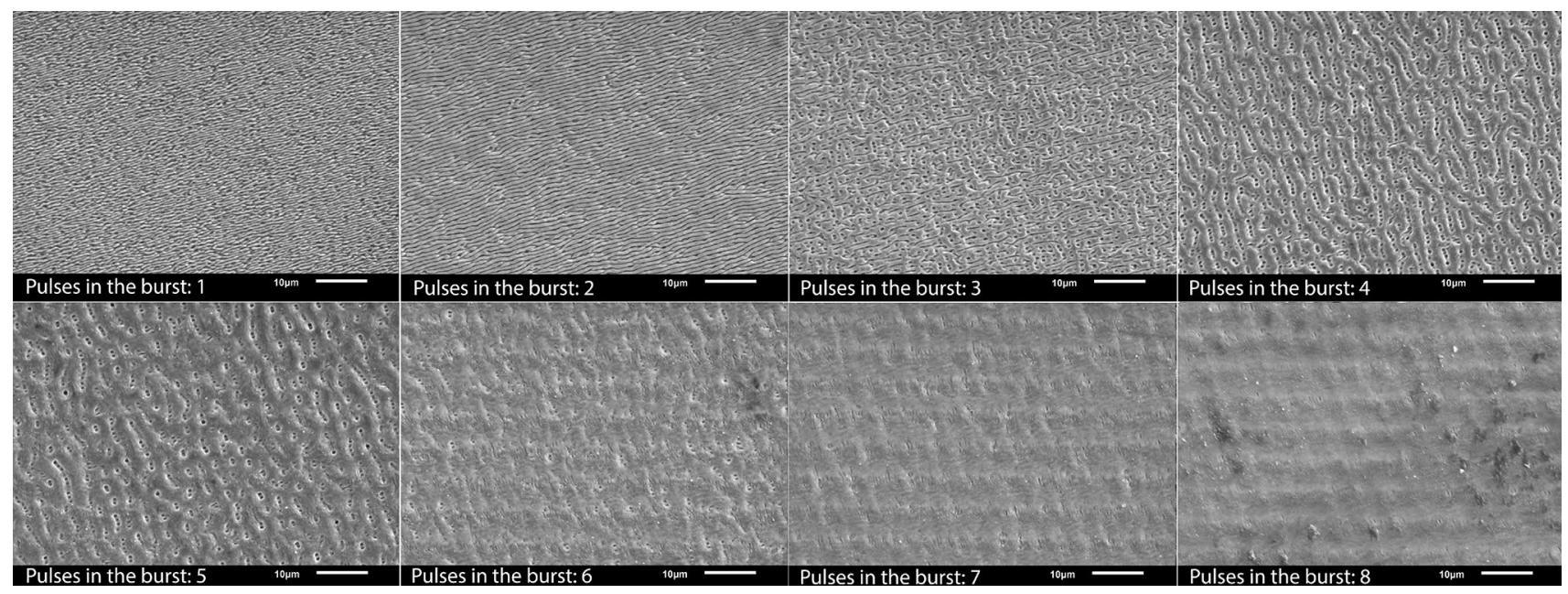

Fig. 11 SEM images of the irradiated TiAlV surface as a function of the number of pulses in the burst at a fluence of $0.2 \mathrm{~J} / \mathrm{cm}^{2}$ per pulse

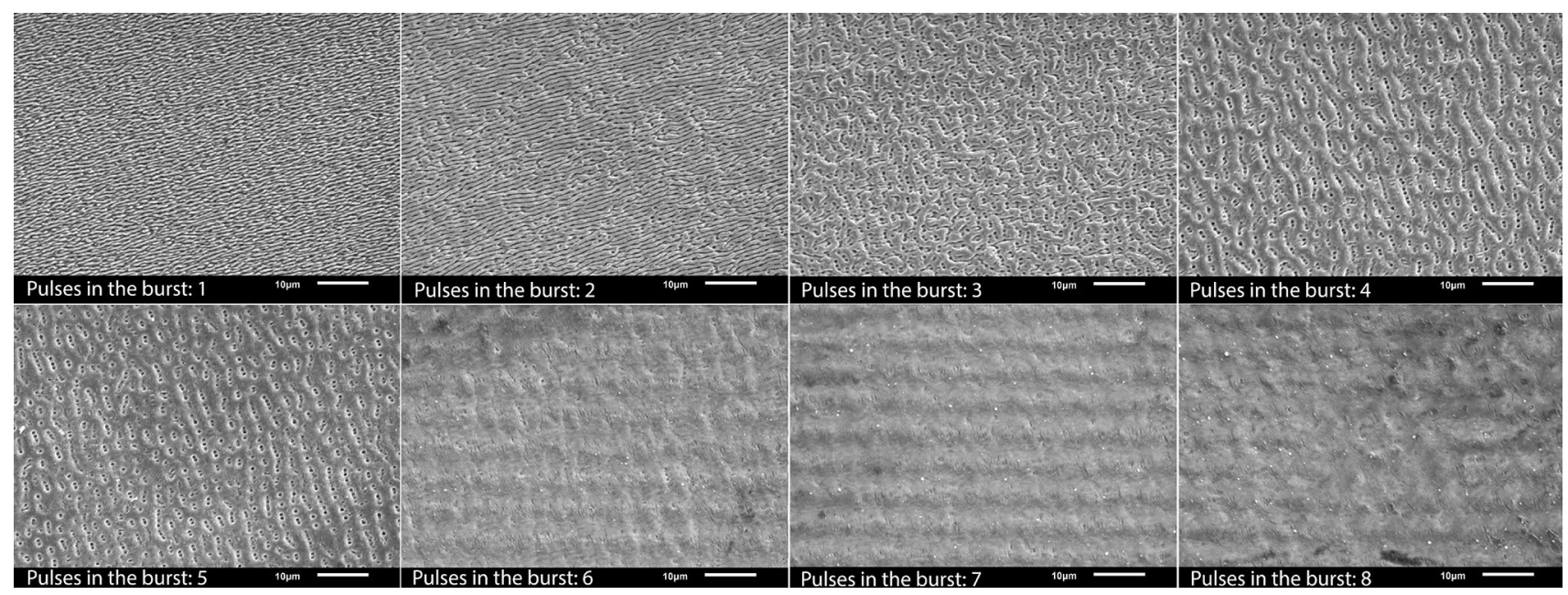

Fig. 12 SEM images of the irradiated TiAlV surface as a function of the number of pulses in the burst at a fluence of $0.25 \mathrm{~J} / \mathrm{cm}^{2}$ per pulse

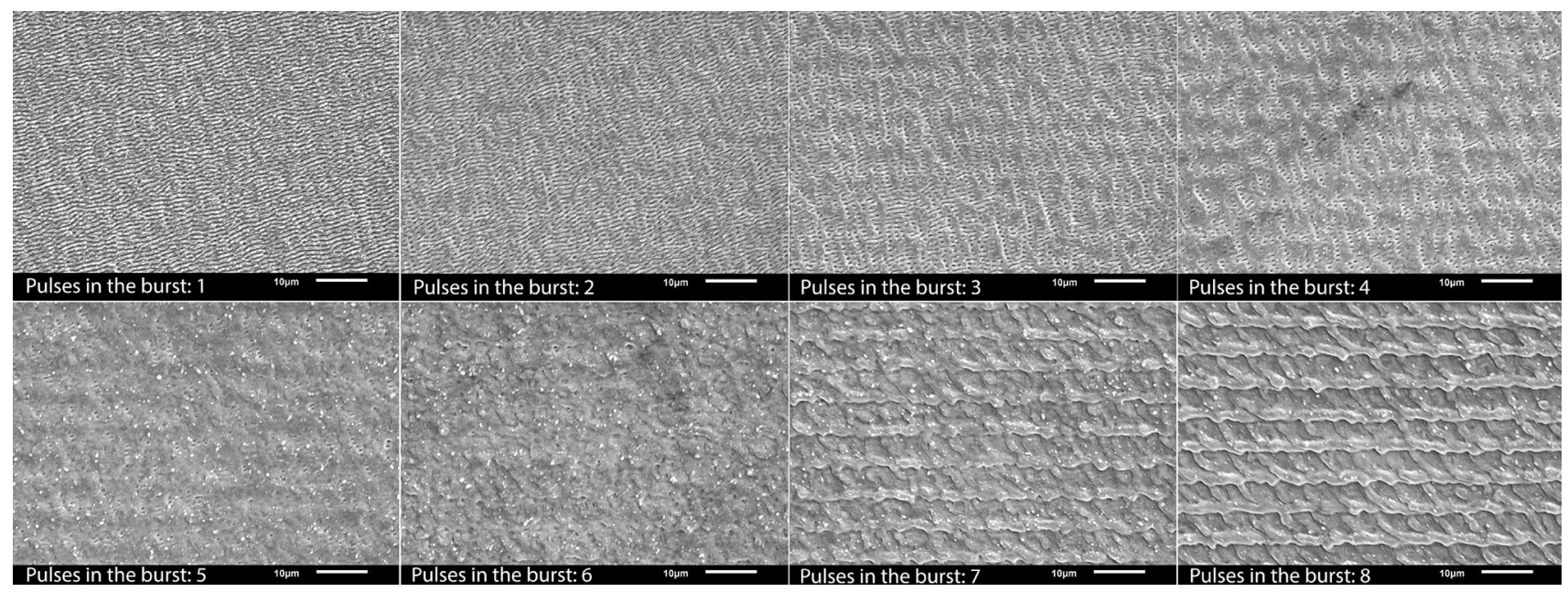

Fig. 13 SEM images of the irradiated TiAlV surface as a function of the number of pulses in the burst at a fluence of $0.5 \mathrm{~J} / \mathrm{cm}^{2}$ per pulse 
when four pulses in the burst are used. In comparison to the corresponding structure depth (see Fig. 4) of the parameter range, where the pore formation on TiAlV occurs and reaches its maximum dimension, it is evident that, analogous to CoCrMo, pores are formed when no material is evaporated. The SEM image in Fig. 14 presents the biggest dimension of the pores in the investigated parameter range with a fluence of $0.25 \mathrm{~J} / \mathrm{cm}^{2}$ per pulse and four pulses in the burst. Compared to the dimension of the pores in CoCrMo (Fig. 9), the pore structure in TiAlV is slightly smaller and the foam structure is thinner. The different dimensions of the pore can be explained in terms of the material-specific thermal conductivities. TiAlV has a thermal conductivity of $6.5 \mathrm{~W} / \mathrm{mK}$ at room temperature [27]. In comparison, the thermal conductivity with $13 \mathrm{~W} / \mathrm{mK}$ of CoCrMo is twice as high [13]. In consequence, a much deeper melt film can be produced in the non-ablation regime of CoCrMo up to the point where evaporation begins and the pore formation decreases again.

\subsection{EDX analysis}

By using the burst mode, heat is accumulated as a result of the high pulse repetition frequency and a melting film with complex melting dynamics is formed. The individual elements of the alloys have different melting and boiling temperatures, therefore the treated area of the material surface is examined by means of an EDX analysis. The results of the qualitative EDX-mapping analysis, presented in Fig. 15 for CoCrMo and in Fig. 16 for TiAlV,

visualize the element distribution in a region of the untreated bulk material (left half of the figure) and the modified region (right half of the figure) where the dimensions of the pores in the respective material are at their maximum.

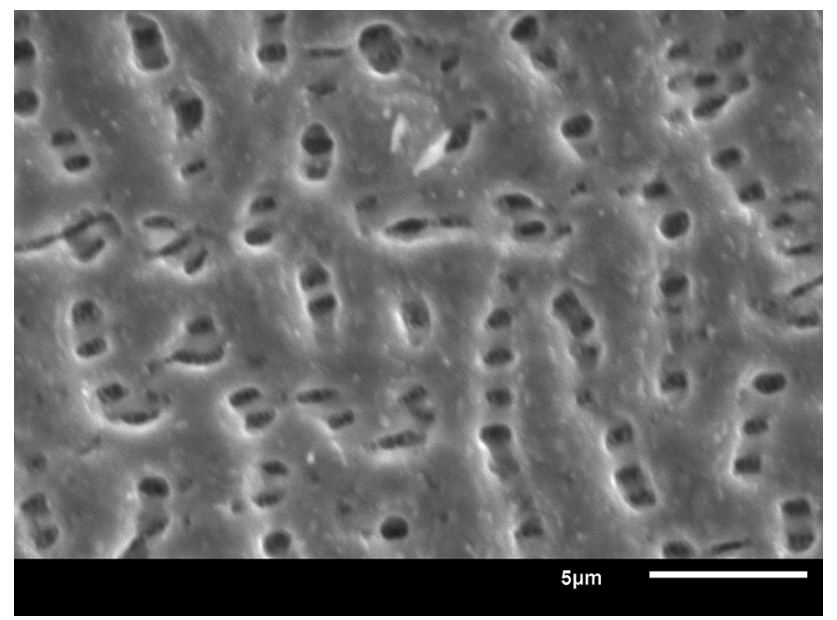

Fig. 14 Pore formation on TiAlV alloy with four pulses in the burst and a fluence of $0.25 \mathrm{~J} / \mathrm{cm}^{2}$ per pulse

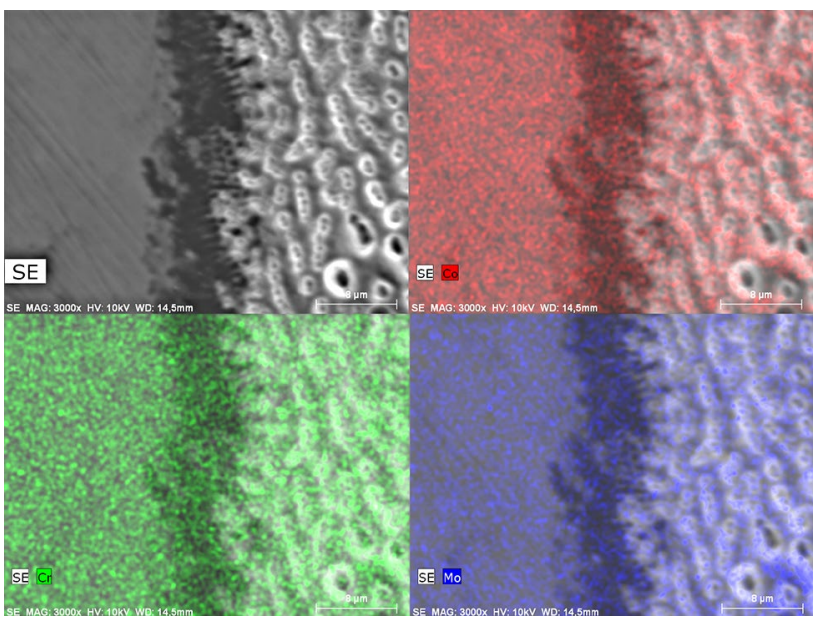

Fig. 15 SEM images of he EDX-Mapping analysis on CoCrMo alloy with an electron acceleration voltage of $10 \mathrm{keV}$. The individual elements refer to the reference picture on the top left. The left halfimage of the individual mapping images represents the untreated bulk material and the right half-image the modified surface with a fluence of $0.5 \mathrm{~J} / \mathrm{cm}^{2}$ per pulse and four pulses in the burst (maximum dimension of the pore formations)

The individual colours represent the concentration of the elements in the respective area. The EDX mapping analysis shows that because of the homogeneous distribution of the main alloy partners over the entire analysis range, the stoichiometry of the material surfaces in CoCrMo and TiAlV tends not to change after the modification in the burst mode. This qualitative result of the EDX mapping is supported quantitatively by the elements considered in the EDX analysis with their concentrations, presented in Table 1 for CoCrMo and 2 for TiAlV.

The two tables also indicate that carbonization of the material surface after pulsed laser modification using burst mode is almost non-existent, even in the parameter range of the maximum dimensions of the pores.

Table 1 Normalized mass concentration of the elements considered in the EDX analysis for CoCrMo on untreated bulk material and on the modified area at a fluence of $0.5 \mathrm{~J} / \mathrm{cm}^{2}$ per pulse and four pulses in the burst

\begin{tabular}{lll}
\hline Element & Bulk $(\%)$ & Modified $(\%)$ \\
\hline Cobalt & $51.74 \pm 1.56$ & $47.14 \pm 1.37$ \\
Chromium & $20.35 \pm 0.61$ & $20.29 \pm 0.61$ \\
Molybdenum & $2.92 \pm 0.13$ & $3.24 \pm 0.15$ \\
Silicon & $0.41 \pm 0.04$ & $0.46 \pm 0.05$ \\
Carbon & $24.58 \pm 2.66$ & $28.87 \pm 3.36$ \\
\hline
\end{tabular}




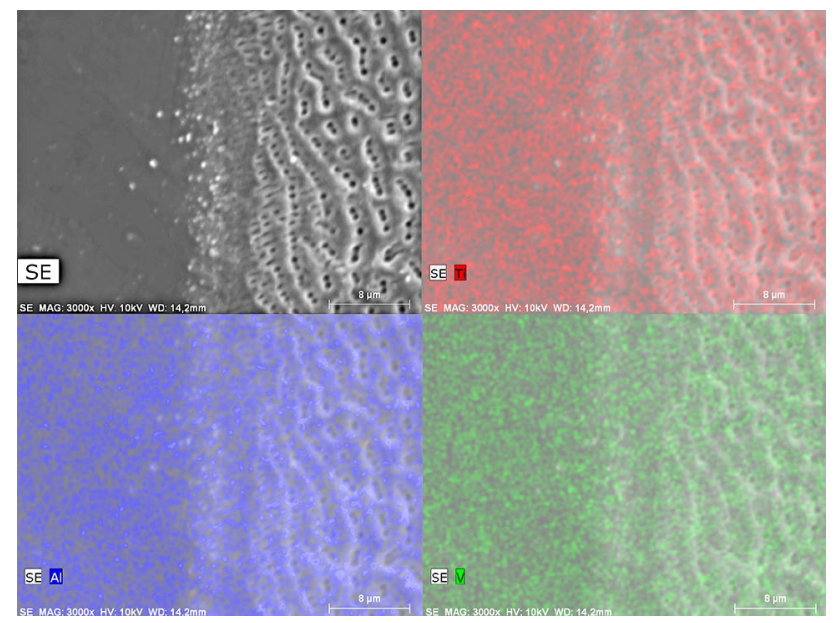

Fig. 16 SEM images of he EDX-Mapping analysis on CoCrMo alloy with an electron acceleration voltage of $10 \mathrm{keV}$. The individual elements refer to the reference picture on the top left. The left halfimage of the individual mapping images represents the untreated bulk material and the right half-image the modified surface with a fluence of $0.25 \mathrm{~J} / \mathrm{cm}^{2}$ per pulse and four pulses in the burst (maximum dimension of the pore formations)

Table 2 Normalized mass concentration of the elements considered in the EDX analysis for TiAlV on untreated bulk material and on the modified area at a fluence of $0.25 \mathrm{~J} / \mathrm{cm}^{2}$ per pulse and four pulses in the burst

\begin{tabular}{lll}
\hline Element & Bulk $(\%)$ & Modified $(\%)$ \\
\hline Titanium & $90.08 \pm 2.90$ & $89.19 \pm 3.52$ \\
Aluminum & $6.29 \pm 0.29$ & $5.43 \pm 0.23$ \\
Vanadium & $1.04 \pm 0.87$ & $2.17 \pm 0.30$ \\
Carbon & $2.39 \pm 0.45$ & $3.21 \pm 0.67$ \\
\hline
\end{tabular}

\section{Summary and outlook}

Results of the laser-induced treatment of cobalt and titanium based alloys by ultrashort pulses with a pulse duration of $10 \mathrm{ps}$ and a wavelength of $1064 \mathrm{~nm}$ in the burst mode were presented. The ablation depth, the surface roughness and the stoichiometry were investigated and discussed in a variation of up to eight pulses in the burst. Regarding to the single pulse ablation threshold of CoCrMo with $0.64 \mathrm{~J} / \mathrm{cm}^{2}$ [13] and TiAlV with $0.37 \mathrm{~J} / \mathrm{cm}^{2}$ [14], the fluence range considered in this work from $0.2 \mathrm{~J} / \mathrm{cm}^{2}$ to $0.75 \mathrm{~J} / \mathrm{cm}^{2}$ per pulse classifies an ablation regime and a non-ablation regime.

Based on the results, it is demonstrated that by using the burst mode as a function of the fluence per pulse and the number of pulses in a burst, a significantly lower but also higher surface roughness can be obtained compared to conventionally pulsed laser radiation (single pulse mode). It was demonstrated that using the burst mode with its high intra-burst pulse repetition frequency of $80 \mathrm{MHz}$ heat is accumulated in the interaction zone, which creates a melt film and the melt dynamics primarily influence the resulting surface roughness. In the ablation regime, the LIPSS generated in the single pulse mode were smoothed at a higher number of pulses in the burst. In the non-ablation regime, a self-organizing, statistically distributed pore system with pore dimensions in the nano- to micrometer range was identified in a parameter range in which no material has been removed by the accumulated heat induced evaporation. An EDX analysis proved that the stoichiometry in the parameter variation, where the dimensions of the pores are maximum, tends not to change.

The realizable dimension of the pore size is comparable to pore formations obtained in other studies, produced by additive methods like plasma chemical oxidation [28, 29], or ablative methods like SLA (sandblasted, large-grit, acidetched) $[30,31]$ and SBF (simulated bubble fluid) [6, 32, 33]. In the above-mentioned studies it was shown that the use of such a pore system significantly improves the growth of bone tissue on endosseous implants. The use of the burst mode on the investigated metallic implant materials offers the possibility to generate a natural, self-organizing pore formation without any measurable material being removed from the surface and without changing the stoichiometry.

As an outlook on further studies, clinical investigations with this patent-pending process will be performed and discussed in upcoming publications. Furthermore, these pores could be used as liquid reservoirs to change the gliding properties of possible functional surfaces.

Acknowledgements The authors gratefully acknowledge financial support by the European Social Fund for Germany (ESF) in the funding project Eila-Sax No. 100339506 and Qualitätsoptimierter Hochrateabtrag No. 100360636.
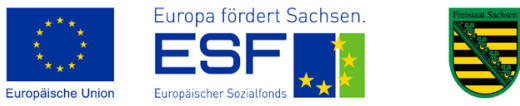

Diese Maßnahme wird mitfinanziert durch Steuermittel auf der Grundlage des vom Sächsischen Landtag beschlossenen
Haushaltes.

Funding Open Access funding enabled and organized by Projekt DEAL.

Open Access This article is licensed under a Creative Commons Attribution 4.0 International License, which permits use, sharing, adaptation, distribution and reproduction in any medium or format, as long as you give appropriate credit to the original author(s) and the source, provide a link to the Creative Commons licence, and indicate if changes were made. The images or other third party material in this article are included in the article's Creative Commons licence, unless indicated otherwise in a credit line to the material. If material is not included in the article's Creative Commons licence and your intended use is not permitted by statutory regulation or exceeds the permitted use, you will 
need to obtain permission directly from the copyright holder. To view a copy of this licence, visit http://creativecommons.org/licenses/by/4.0/.

\section{References}

1. L. Gemini, M. Faucon, L. Romoli, R. Kling, in Laser-based Micro-and Nanoprocessing XI (International Society for Optics and Photonics, 2017)

2. D. Sierra, S. Edwardson, G. Dearden, Procedia CIRP 74, 362 (2018). https://doi.org/10.1016/j.procir.2018.08.143

3. X. Hao, H. Li, X. Song, L. Li, N. He, J. Micro Nanomanuf. 6, 021001 (2017). https://doi.org/10.1115/1.4038629

4. J. Yong, Q. Yang, C. Guo, F. Chen, X. Hou, RSC Adv. 9, 12470 (2019). https://doi.org/10.1039/C8RA10673H

5. A. Vogel, N. Linz, S. Freidank, G. Paltauf, Phys. Rev. Lett. 100(3), 038102 (2008). https://doi.org/10.1103/PhysRevLett.100.038102

6. H. Wang, C. Liang, Y. Yang, C. Li, Biomed. Mater. 5(5), 054115 (2010). https://doi.org/10.1088/1748-6041/5/5/054115

7. J. Bonse, J. Krüger, S. Höhm, A. Rosenfeld, J. Laser Appl. 24(4), 042006 (2012). https://doi.org/10.2351/1.4712658

8. S. Rung, K. Bokan, K. Rutsch, S. Schwarz, C. Esen, R. Hellmann, J. Laser Micro Nanoeng. 13, 160 (2018). https://doi.org/10.2961/ jlmn.2018.03.0004

9. J. Bonse, S. Höhm, S. Kirner, A. Rosenfeld, J. Krüger, in Conference on Lasers and Electro-Optics (Optical Society of America, 2016), p. STh1Q.3. https://doi.org/10.1364/CLEO_SI.2016. STh1Q.3

10. F. Müller, C. Kunz, S. Gräf, Materials 9, 476 (2016). https://doi. org/10.3390/ma9060476

11. J. Skolski, G. Römer, A. Veld, V. Mitko, J.V. Obona, V. Ocelik, J.T.M. De Hosson, J. Laser Micro/Nanoeng. 5, 3 (2010). https:// doi.org/10.1007/s00170-015-6831-x

12. S. Nolte, F. Schrempel, F. Dausinger, Springer Ser. Opt. Sci. 195, 200 (2016). https://doi.org/10.1007/978-3-319-17659-8

13. D. Metzner, P. Lickschat, S. Weißmantel, Appl. Phys. A 126(2), 84 (2020). https://doi.org/10.1007/s00339-019-3203-7

14. J. Cheng, W. Perrie, M. Sharp, S. Edwardson, N. Semaltianos, G. Dearden, K. Watkins, Appl. Phys. A 95(3), 739 (2009). https:// doi.org/10.1007/s00339-008-5037-6

15. F.D. Niso, C. Gaudiuso, T. Sibillano, F.P. Mezzapesa, A. Ancona, P.M. Lugarà, Opt. Express 22(10), 12200 (2014). https://doi. org/10.1364/OE.22.012200

16. D. Metzner, P. Lickschat, S. Weißmantel, Appl. Phys. A 125(7), 3439 (2019). https://doi.org/10.1007/s00339-019-2755-x

17. J. Mazumder, Opt. Eng. 30(8), 1208 (1991). https://doi. org/10.1117/12.55899
18. C. Chan, J. Mazumder, M. Chen, Metall. Trans. A 15(12), 2175 (1984). https://doi.org/10.1007/BF02647100

19. J.M. Drezet, S. Pellerin, C. BezençSon, S. Mokadem, Journal de Physique IV (Proceedings) (2004). https://doi.org/10.1051/ jp4:2004120034

20. S. Das, S. Kar, Mater. Sci. Forum 978, 34 (2020). https://doi. org/10.4028/www.scientific.net/MSF.978.34

21. K. Setoura, S. Ito, H. Miyasaka, Nanoscale 90, 719 (2017). https ://doi.org/10.1039/C6NR07990C

22. S. O'Shaughnessy, A. Robinson, Ann. N. Y. Acad. Sci. 1161, 304 (2009). https://doi.org/10.1111/j.1749-6632.2008.04332.x

23. M. Gouge, J. Heigel, P. Michaleris, T. Palmer, Int. J. Adv. Manuf. Technol. (2015). https://doi.org/10.1007/s00170-015-6831-x

24. D. Metzner, P. Lickschat, S. Weißmantel, Appl. Phys. A 125(6), 172 (2019). https://doi.org/10.1007/s00339-019-2696-4

25. B. Neuenschwander, B. Jaeggi, D.J. Foerster, T. Kramer, S. Remund, J. Laser Appl. 31(2), 022203 (2019). https://doi. org/10.2351/1.5096083

26. P. Lickschat, A. Demba, S. Weissmantel, Appl. Phys. A 123(2), 137 (2017). https://doi.org/10.1007/s00339-016-0743-y

27. W. Pan, S. Ding, J. Mo, Int. J. Adv. Manuf. Technol. 75(5-8), 1077 (2014). https://doi.org/10.1007/s00170-014-6094-y

28. M. Diefenbeck, T. Mückley, C. Schrader, J. Schmidt, S. Zankovych, J. Bossert, K.D. Jandt, M. Faucon, U. Finger, Biomaterials 32(32), 8041 (2011). https://doi.org/10.1016/j.biomateria 1s.2011.07.046

29. M. Diefenbeck, C. Schrader, F. Gras, T. Mückley, J. Schmidt, S. Zankovych, J. Bossert, K.D. Jandt, A. Völpel, B.W. Sigusch, H. Schubert, S. Bischoff, W. Pfister, B. Edel, M. Faucon, U. Finger, Biomaterials 101, 156 (2016). https://doi.org/10.1016/j.biomateria 1s.2016.05.039

30. S. Roehling, B. Meng, D. L. Cochran, Implant Surfaces and their Biological and Clinical Impact pp. 93-136 (2015). https://doi. org/10.1007/978-3-662-45379-7_9

31. G.L. Stafford, Evidence Based Dentistry 15, 87 EP (2014). https ://doi.org/10.1038/sj.ebd.6401047

32. E. Pecheva, T. Petrov, C. Lungu, P. Montgomery, L. Pramatarova, Chem. Eng. J. 137(1), 144 (2008). https://doi.org/10.1016/j. cej.2007.07.096

33. Y. Yang, J. Yang, C. Liang, H. Wang, X. Zhu, N. Zhang, Opt. Exp. 17(23), 21124 (2009). https://doi.org/10.1364/OE.17.021124

Publisher's Note Springer Nature remains neutral with regard to jurisdictional claims in published maps and institutional affiliations. 